\title{
Shubnikov-de Haas oscillations, peaks and different temperature regimes of the diagonal conductivity in the integer quantum Hall conductor
}

\author{
V.M. Gvozdikov \\ Kharkov National University, 61077, Kharkov, Ukraine \\ Max-Planck-Institut für Physik Komplexer Systeme, \\ Nöthnitzer Strasse 38, D-01187 Dresden, Germany
}

Received April 27, 2005

\begin{abstract}
A theory for the Shubnikov - de Haas oscillations in the diagonal conductivity $\sigma_{x x}$ of a 2D conductor is developed for the case when electron states within the broaden Landau levels are localized except the narrow stripe in the center. The standard Shubnikov-de Haas oscillations take place only in the low-field region which at higher magnetic fields crosses over into peaks. In the limit $\Omega \tau \gg>1$ peaks in the $\sigma_{x x}$ became sharp and between them $\sigma_{x x} \rightarrow 0$ ( $\Omega$ is the cyclotron frequency, $\tau$ is the electron scattering time). The conductivity peaks display different temperature behavior with the decrease of temperature, $T$ : a thermal activation regime, $\sigma_{x x} \propto \exp (-\Delta / T)$, which holds at higher temperatures, crosses over into the variable-range-hopping regime at lower temperatures with $\sigma_{x x} \propto 1 / T \exp \left(-\sqrt{T_{0} / T}\right)$ (the prefactor $1 / T$ is absent in the conductance).
\end{abstract}

PACS: 73.43.-f, 73.40.Gk, 75.47.-m

In spite of more than two decades of intensive experimental research and large theoretical efforts, the quantum magnetic oscillations of the conductivity in $2 \mathrm{D}$ conductors still have some open questions. Even in the most studied case of the integer quantum Hall effect (IQHE) [1] a complete picture is missing, in particular, concerning different regimes in temperature and magnetic field dependencies of the Hall, $\sigma_{x y}$, and longitudinal, $\sigma_{x x}$, conductivities. Although the origin of the quantized plateaus in the $\sigma_{x y}$ is well understood [2] the transitional regions between them, where localization and scaling [3] play an important role, needs a deeper insight. The scaling properties of diagonal conductivity $\sigma_{x x}$ in the variable-range hopping (VRH) regime of an IQHE were recently established experimentally at low temperatures down to $60 \mathrm{mK}$ in [4]. To explain this universal scaling behavior as well as transitions between different regimes in the IQHE is a theoretical challenge. At the moment there is no coherent analytic description of the quantum magnetic oscillations of the diagonal conductivity $\sigma_{x x}$ which takes into account the localization effects at different temperature regimes in the IQHE. In particular, it is not clear so far why quantum oscillations in $\sigma_{x x}$ do survive in spite of the fact that most states within the broaden Landau levels (LL) are localized (i); Why $\sigma_{x x} \rightarrow 0$ between the peaks in the limit $\Omega \tau \gg>1$, if at low fields it displays a standard Shubnikov - de Haas ( $\mathrm{SdH}$ ) oscillations (ii); Why with the decrease of temperature, $T$, the peaks in $\sigma_{x x}$ display first a thermal activation behavior $\sigma_{x x} \propto \exp (-\Delta / T)$, which then crosses over into the VRH regime at low temperatures with $\sigma_{x x} \propto 1 / T \exp \left(-\sqrt{T_{0} / T}\right)$ (iii); Why the prefactor $1 / T$ is absent in the conductance (iv).

The well established fact is that localization in the IQHE picture plays a crucial role. On the other hand, nonzero conductivity is impossible without the extended states. It is believed that extended states are within the narrow stripe at the center of the broaden LL and all the other states are localized [5]. One can not give a simple physical picture for these localized states in general. At high fields the presence of the localized states in the 2D conductor means that Landau orbits drift along the closed equipotential contours of the impurity potential. At places where contours come close electrons can tunnel from one contour to another providing thereby a conductivity mechanism through the extended states. The diagonal conductivity $\sigma_{x x}$ and the Hall conductivity $\sigma_{x y}$ are closely related in the IQHE. The peaks in the $\sigma_{x x}$ are exactly at the same fields where $\sigma_{x y}$ transits from one plateau to another. An ideal picture of the 
IQHE at $T=0$ assumes that $\sigma_{x x}=0$ within the plateaus while the $\sigma_{x y}=n e^{2} / h$ is quantized ( $n$ is an integer). In real experiments $T \neq 0$ and $\sigma_{x x} \neq 0$. In the low-field region $\sigma_{x x}$ displays the $\mathrm{SdH}$ oscillations and plateaus in the $\sigma_{x y}$ are unresolved.

In this Letter a theory is developed for the magnetic quantum oscillations of the $\sigma_{x x}$ in $2 \mathrm{D}$ conductors with localization which explains properties (i)-(iv). The conductivity mechanism in this theory is due to the electron hopping between Landau orbits within the conducting plane. A similar mechanism for the conductivity due to the electron tunneling between Landau orbits from the neighboring layers was considered in [6] for the case of incoherent electron hopping across the layers in organic conductor. This hopping mechanism remains in effect if the Landau orbits lay within the same conducting plane or belong to the different tunnel-coupled 2D conductors. The latter is important in view of recent observation of a typical IQHE behavior in the tunneling conductance of a two coupled Hall bars reported in [7]. The tunneling conductance in this experiment displays the same scaling features as those usually observed in the bulk Hall sample and, therefore, can not be explained by the tunneling between the two counter-propagating edge states. On the other hand, electron tunneling between Landau orbits from different Hall bars and within the same sample equally contribute into the total conductance. In view of that one can anticipate the standard IQHE behavior in the tunneling conductance observed in experiment [7].

According to [6] the tunneling $\mathrm{SdH}$ conductivity can be written as a sum of the Boltzmann $\left(\sigma_{B}\right)$ and quantum $\left(\sigma_{Q}\right)$ terms: $\sigma_{x x}=\sigma_{B}+\sigma_{Q}$, where

$$
\begin{aligned}
\sigma_{B} & =\sigma_{0} \int d \varepsilon \frac{d E}{\pi} g(\varepsilon) v_{x}^{2}(\varepsilon)\left(-\frac{\partial f}{\partial E}\right) \tau S[\lambda, \delta(E, \varepsilon)], \\
\sigma_{Q} & =\sigma_{0} \int d \varepsilon \frac{d E}{\pi} g(\varepsilon) v_{x}^{2}(\varepsilon)\left(\frac{\partial f}{\partial E}\right) \frac{2 \pi}{\Omega} \frac{\partial}{\partial \lambda} S[\lambda, \delta(E, \varepsilon)] .
\end{aligned}
$$

Here $\lambda(E)=2 \pi / \Omega \tau, \delta(E, \varepsilon)=2 \pi(E+\varepsilon) / \hbar \Omega, \sigma_{0}=$ $=e^{2} N_{L} / \hbar \Omega, N_{L}=\Phi / S \Phi_{0}$ is the electron density at the LL, $\Phi$ is the flux through a sample, $\Phi_{0}=\hbar c / 2 \pi e$, and

$$
S(\lambda, \delta)=\sum_{p=-\infty}^{\infty}(-1)^{p} \mathrm{e}^{-|p| \lambda} \cos p \delta=\frac{\sinh \lambda}{\cosh \lambda+\cos \delta} .
$$

The variable $\varepsilon$ describes the LLs broadening by impurities with the density of states (DOS) $g(\varepsilon)$ :

$$
E_{n}(\varepsilon)=\hbar \Omega(n+1 / 2)+\varepsilon .
$$

The electron velocity $v_{x}$ is related to the tunneling matrix elements by [6]

$$
v_{x}(\varepsilon)=\frac{\left|t_{\varepsilon, \varepsilon}\right| R}{\hbar \sqrt{2}},
$$

where $R$ and $\hbar \sqrt{2} /\left|t_{\varepsilon, \varepsilon}\right|$ are correspondingly the distance and the time of tunneling. A strong point of the above equations is that we can learn much about the $\sigma_{x x}(B, T)$ without resort to the specific models for the localization ( $B$ is the magnetic field). In any such model the $g(\varepsilon)$ has a narrow band of delocalized states where the $v_{x}(\varepsilon) \neq 0$. It is generally accepted [5] that only one state, precisely at the LL $(\varepsilon=0)$, is delocalized. For the localized states $v_{x}(\varepsilon)=0$. Thus, only one level, $\varepsilon=0$, or a narrow stripe of delocalized states, contribute into Eqs. (1), (2).

The scattering time $\tau$ in general is a model-dependent function of the energy which is inversely proportional to the scattering probability for the conducting (delocalized) electrons. The latter belong to a narrow stripe in the $g(\varepsilon)$ while the rest of electrons are localized and produce a reservoir of states stabilizing oscillations in $\tau$. Thus, we can put $\tau=$ const in Eqs. (1), (2), which yield:

$$
\begin{gathered}
\sigma_{x x}=\sigma_{\tau} \int \frac{d E}{\pi}\left(-\frac{\partial f}{\partial E}\right)\left[G_{B}(\lambda, E)+G_{Q}(\lambda, E)\right], \\
G_{B}(\lambda, E)=S[\lambda, \Delta(E)], \\
G_{Q}(\lambda, E)=-\lambda \frac{\partial}{\partial \lambda} S[\lambda, \Delta(E)]=-\lambda \frac{1+\cosh \lambda \cos \Delta}{(\cosh \lambda+\cos \Delta)^{2}},
\end{gathered}
$$

where $\Delta(E)=2 \pi E / \hbar \Omega$ and

$$
\sigma_{\tau}=\frac{e^{2} N_{L} \tau\left\langle v_{x}^{2}\right\rangle}{\hbar \Omega}
$$

The average of the velocity squared, is given by

$$
\left\langle v_{x}^{2}\right\rangle=\frac{R^{2}}{2 \hbar^{2}} \int_{\varepsilon_{\min }}^{\varepsilon_{\max }} d \varepsilon g(\varepsilon)\left|t_{\varepsilon, \varepsilon}\right|^{2} .
$$

The integral in (10) is taken within the narrow stripe of the delocalized states. The functions $G_{B}(\lambda, E)$ and $G_{Q}(\lambda, E)$ are sharply peaked at $E=E_{n}$ and between the LLs they nearly compensate each other, as shown in Fig. 1. This demonstrates clearly that the Boltzmann term alone, $G_{B}(\lambda, E)$, is insufficient for the correct description and only by taking account of the quantum term, $G_{Q}(\lambda, E)$, one can explain why $\sigma_{x x}$ tends to zero between the peaks in the IQHE. The width of peaks in Fig. 1 in the energy scale is of the order of $\hbar / \tau$. If $T>>\hbar / \tau$, then the peaked function 

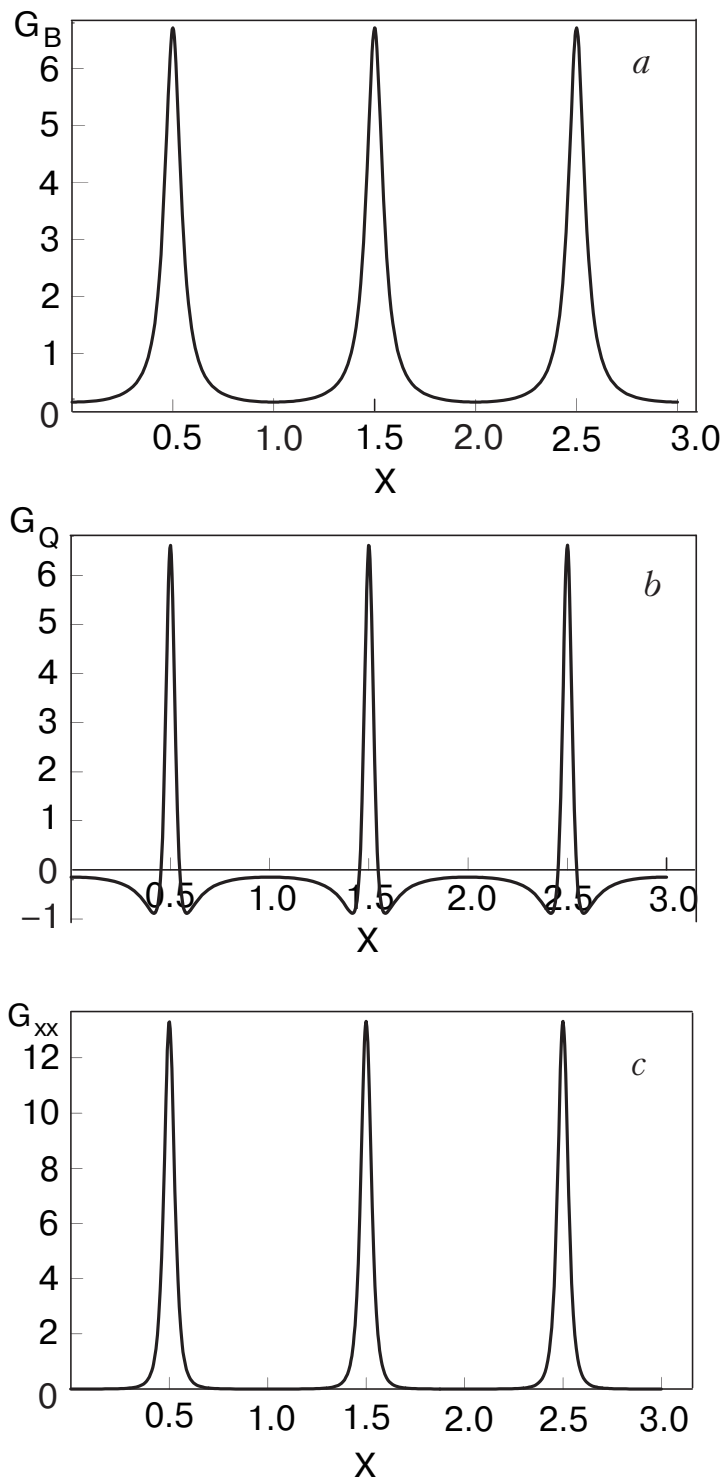

Fig. 1. The Boltzmann, $G_{B}=S(\lambda, 2 \pi X)$ (Fig. 1,a), and the quantum, $G_{Q}=-\lambda \partial S(\lambda, 2 \pi X) / \partial \lambda$ (Fig. 1,b), contributions into the conductivity $\sigma_{x x}$ in Eq. (6), and their sum $G_{x x}=G_{B}+G_{Q}($ Fig. 1,c). $X=E / \hbar \Omega, \lambda=0.3$.

$-\partial f / \partial E$ is broader than, the $G_{x x}(\lambda, E)=G_{B}(\lambda, E)+$ $+G_{Q}(\lambda, E)$, and we can approximate the $G_{x x}(\lambda, E)$ in Eq. (6) by

$$
G_{x x}(\lambda, E) \approx \frac{2}{\pi} \sum_{n=-\infty}^{\infty} \frac{\eta}{(n+1 / 2-E / \hbar \Omega)^{2}+\eta^{2}},
$$

where $\eta=\lambda / 2 \pi$. For $\eta<<1$ Eq. (11) can be easily proved analytically with the help of the identity [2]

$$
\frac{1}{\pi} \sum_{p=-\infty}^{\infty} \frac{\eta}{(n+a)^{2}+\eta^{2}}=\frac{\sinh 2 \pi \eta}{\cosh 2 \pi \eta-\cos 2 \pi a} .
$$

Thus, for high temperatures, $T>\hbar / \tau$, we have

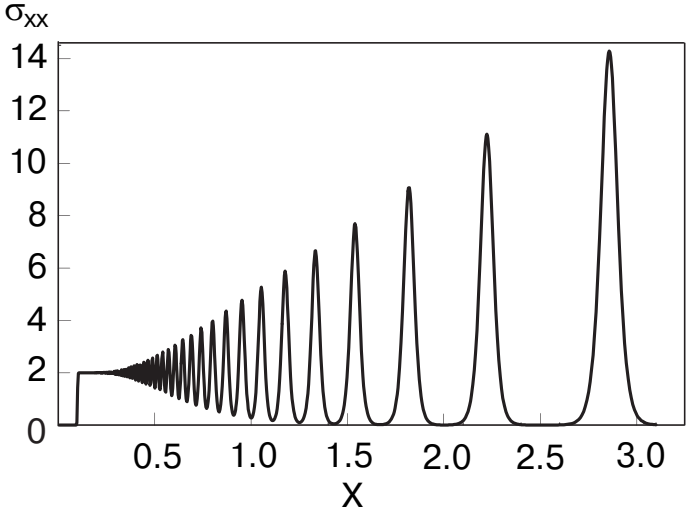

Fig. 2. The conductivity $\sigma_{x x}$ [see Eq. (13)] in units of $\sigma_{\tau}$ as a function of the $X=\hbar \Omega$. The conventional energy units accepted in which $T=0.2$ and $E_{F}=10$.

$$
\sigma_{x x}(B) \approx \sigma_{\tau} \frac{\hbar \Omega}{4 \pi T} \sum_{n} \cosh ^{-2}\left(\frac{E_{n}-\mu}{T}\right) .
$$

This sharply-peaked function of the $\hbar \Omega$ is shown in Fig. 2. The same function describes the quantum magnetic oscillations of the ultrasound absorption in metals [8]. A temperature dependence of the peaks in $\sigma_{x x}(B)$ for different temperatures is plotted in Fig. 3.

Under the condition $\hbar \Omega / T>>1$, the conductivity $\sigma_{x x}$ at maxima (i.e., when $E_{n}=\mu$ ) is given by $\sigma_{x x}=\sigma_{\tau} \hbar \Omega / 4 \pi T$. At minima (i.e., when the chemical potential $\mu$ falls between the LL) the conductivity $\sigma_{x x}$ is exponentially small:

$$
\sigma_{x x}=\sigma_{\tau} \frac{\hbar \Omega}{4 \pi T} \exp \left(-\frac{\hbar \Omega-E_{0}}{T}\right)
$$

( $E_{0}$ is a position of the $\mu$ between the LL). Such an activation dependence is well established in the $\sigma_{x x}(T)$ in the IQHE regime [5]. At lower temperatures, $T<<\hbar / \tau$, one can approximate the $-\partial f / \partial E$ by $\delta(E-\mu)$, to obtain

$$
\sigma_{x x} \approx \sigma_{\tau}\left(G_{B}[\lambda, \Delta(\mu)]+G_{Q}[\lambda, \Delta(\mu)]\right) .
$$

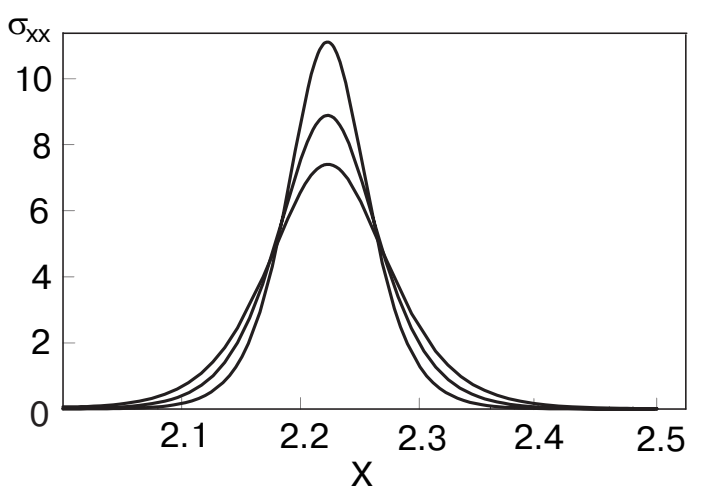

Fig. 3. The same as in Fig. 2 for three different temperatures $T=0.2,0.25$, and 0.3 (from top to bottom). 
The $\sigma_{x x}$ in (14) is sharply peaked function of the $\Delta(\mu)=2 \pi \mu / \hbar \Omega$ as shown in Fig. 1. The Boltzmann and the quantum terms in Eq. (14) nearly compensate each other between the peaks which in the limit $\eta \rightarrow 0$ become narrow Lorentzians of Eq. (11). The temperature dependence of the $\sigma_{x x}$ at $T<<\hbar / \tau$ in Eq. (14) comes only from the $\sigma_{\tau}$ due to the VRH mechanism. The VRH concept in the IQHE problem is now well established [4,9]. It was introduced in [10] and well describes the scaling properties of the peaks in the $\sigma_{x x}$ within the plateau-to-plateau transition region. The diagonal and the Hall conductivities in this region are related by the «semicircle» law [11]. In samples with mobilities $\sim 10^{6} \mathrm{~cm}^{2} /(\mathrm{V} \cdot \mathrm{s})$ at a few tens $\mathrm{mK}$ the best experimental fit yields [4]:

$$
\sigma_{\tau}=\frac{A}{T} \exp \left(-\sqrt{T_{0} / T}\right) .
$$

The above equation describes a well-known Mott hopping conductivity [12] in which the square root $\sqrt{T_{0} / T}$ in the exponent means that the system is a one-dimensional. For two and three dimensions there should be $\left(T_{0} / T\right)^{\alpha}$ with $\alpha=1 / 3$ and $1 / 4$, correspondingly. Since the system in question is a $2 \mathrm{D}$, the authors of the [10] derived Eq. (15) with $\alpha=1 / 2$ assuming a strong Coulomb interaction between electrons. In that case the temperature $T_{0}=C e^{2} /(4 \pi \epsilon \xi)$ is proportional to the Coulomb energy at the localization length $\xi(v), \in$ is the dielectric constant, and $C \sim 1$. Numerous experiments and calculations testify in favor of a universal (i.e., independent on the Landau-level index) critical behavior of the localization length $\xi(v) \propto\left|v-v_{c}\right|^{-\gamma}$ near the LLs [2,3,5]. Here $v=N \Phi_{0} / B$ is the filling factor, $v_{c}$ is the critical filling factor, and $\gamma \approx 2.35$ is a universal critical exponent. The divergency of the $\xi(v)$ at $v_{c}$ means that this is a critical point for the transition from the dielectric to the conducting state.

The explanation of the $\alpha=1 / 2$ in Eq. (15) by the Coulomb interaction is inconsistent at least for the case of IQHE which is known to be a free-electron phenomenon. The coherence length as well is calculated for free electrons without Coulomb interaction. Also, this approach encounters with some difficulties in the experimental interpretations, like, for example, a divergency of the dielectric constant at the critical point [13].

On the other hand, in our approach Eq. (15) directly follows from (9) and (10). In the spirit of the VRH concept, we can estimate the $\left|t_{\varepsilon, \varepsilon}\right|^{2}$ as a quantity proportional to the electron hopping probability between the two 1D closed equipotential contours at which Landau orbits are localized. If $R$ is a distance of hopping, then

$$
\left|t_{\varepsilon, \varepsilon}\right|^{2} \propto \exp \left[-\left(\frac{1}{R N(0) T}+\frac{2 R}{\xi}\right)\right] .
$$

In this equation we take account of the thermal activation which helps the tunneling if the initial and final levels are separated in energy by a stripe of the order of $1 / R N(0)$. Here $N(0)$ is some one-dimensional DOS at the closed equipotential contours averaged over a random potential and taken near the Fermi level. The optimal hopping distance is $R=\sqrt{\xi / 2 N(0) T}$ and Eqs. (10),(16) yield $\left\langle v_{x}^{2}\right\rangle \propto 1 / T \exp \left(-\sqrt{T_{0} / T}\right)$. Therefore, in view of Eq. (9) and that $T_{0}=8 / N(0) \xi$, we arrive at the VRH conductivity (15). In fact, the quantity $T_{0}$ is a fitting parameter which can be found only from the experiment. The quantity $N(0)$ determines the average energy separation (or the gap) between the initial an final states in the electron hopping. In the case when the Coulomb repulsion between electrons plays a dominant role this gap can can be estimated as $C e^{2} /(4 \pi \epsilon R)$ which yields $T_{0}=C e^{2} /(4 \pi \epsilon \xi)$ as in [10]. By taking effective DOS in the form $1 / N_{\text {eff }}=$ $=1 / N(0)+C e^{2} /(4 \pi \epsilon)$ one can qualitatively take account of the Coulomb effect in the Mott hopping mechanism. We see that the resulting VRH conductivity is the same as without Coulomb interaction with the only difference in the DOS N(0) $\rightarrow N_{\text {eff }}$ which is unimportant since the DOS is a fitting parameter. In the IQHE regime Coulomb interaction does not play any significant role and can be discarded without change in the shape of the hopping conductivity given by Eq. (15).

The VRH concept based on this equation was originally applied to the problem of the conductivity peak broadening $\Delta v$ in [10]. It was shown that the temperature, current, and frequency dependencies of the $\Delta v$ can be well described within this paradigm. Here we derived a prefactor $A / T$ which also have been observed in the $\sigma_{x x}(T)[4,9]$. However, it should be noted that the prefactor $A / T$ is absent in the experiments in which a conductance was measured [14,15]. The difference is because the conductivity in Eqs. (1), (2) is proportional to the $v_{x}^{2} \propto R^{2} \propto 1 / T$. The conductance $\sigma_{x x}^{c}(T) \propto\left(e^{2} / \hbar\right)\left|t_{\varepsilon, \varepsilon}\right|^{2}$ and has no factor $R^{2} \propto 1 / T$. Therefore, at the same conditions as in Eq. (14) the conductance is

$$
\begin{gathered}
\sigma_{x x}^{c} \approx \sigma_{\tau}^{c}\left(G_{B}[\lambda, \Delta(\mu)]+G_{Q}[\lambda, \Delta(\mu)]\right), \\
\sigma_{\tau}^{c}=A_{c} \exp \left(-\sqrt{T_{0} / T}\right) .
\end{gathered}
$$

Since $T_{0} \propto 1 / \xi \propto\left|v-v_{c}\right|^{\gamma}$ the function $\sigma_{\tau}^{c}(v)$ has a fixed maximum value $\sigma_{\tau}^{c}=A_{c}$ at $v=v_{c}$ for different temperatures. This remarkable property of the conductance is firmly established in the VRH regime at low temperatures $[11,14,15]$. 
So far we assumed that the chemical potential is a constant. In $2 \mathrm{D}$ conductors $\mu(B)$ is an oscillating function [14] satisfying the equation [6]:

$$
\mu=E_{F} \pm \frac{\hbar \Omega}{\pi} \arctan \left[\frac{\sin (2 \pi \mu / \hbar \Omega)}{\mathrm{e}^{v}+\cos (2 \pi \mu / \hbar \Omega)}\right] .
$$

The sign $(-)$ here stands for the direct and $(+)$ for the inverse sawtooth. The amplitude of these oscillations is of the order of the $\hbar \Omega$ which is small compared to the Fermi energy $E_{F}$. It was shown in [6] that in a quasi 2D layered conductor the peaks in the magnetic conductivity across the layers are split in the case $\mu(B)$ is an inverse sawtooth function. The very same effect holds for the $\sigma_{x x}$, as shown in Fig. 4, which displays the $\sigma_{x x}(B)$ according to Eq. (14) with the
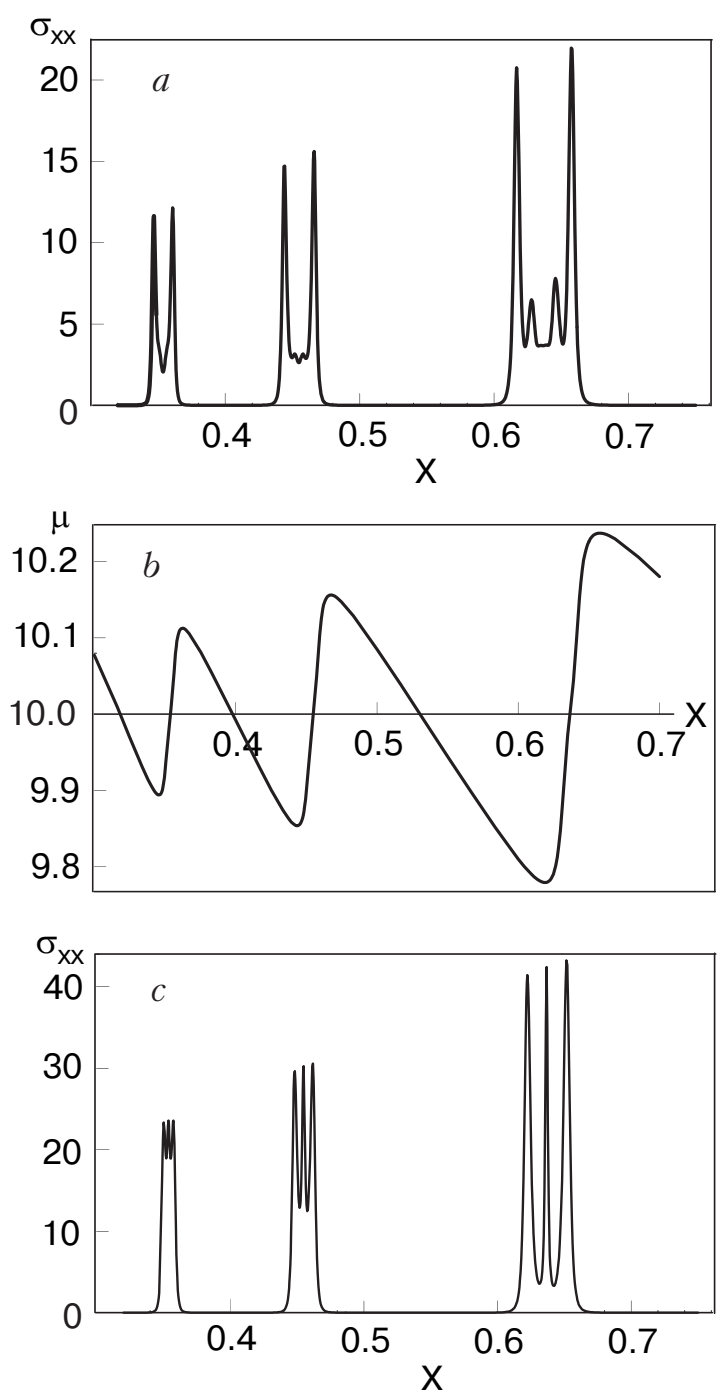

Fig. 4. The conductivity $\sigma_{x x}(X)$ given by Eq. (14) (Fig. $4, a)$ in which the chemical potential $\mu(X)$ oscillates as in Fig. 4,b (a direct sawtooth, see Eq. (17) and text). The choice of the units is the same as in Fig. 2. The spin-splitting parameter $s=0.093, E_{F}=10, v=0.06$, and $X=\hbar \Omega$. In Fig. 4,c $s=0$.
$\mu(B)$ given by Eq. (19). We also take account of the spin-splitting which is easy to incorporate by the substitution $\mu \rightarrow \mu \pm \mu_{e} B$ into the right-hand side of Eq. (19) and average it over two spin configurations ( $\mu_{e}$ is the magnetic moment of electron). The spin-splitting parameter $s=2 \pi \mu_{e} B / \hbar \Omega$ can be rewritten in terms of the $g$ factor and the effective mass to the electron mass ratio $s=\pi g m^{*} / m$. In GaAs $g \approx 0.44$ and $m^{*} / m \approx 0.068$ which yield $s \approx 0.093$. This value gives a pronounced splitting in the peaks in Fig. 4, $a$, but it is much less noticeable in Fig. $4, b$ for $\mu(B)$. The shape of peaks in the absence of splitting $(s=0)$ is shown in Fig. 4,c. The origin of correlations between the shapes of the function $\sigma_{x x}(B)$ and the $\mu(B)$ is the same as in layered conductors [6].

In conclusion, we suggested a model of the hopping conductivity which describes different regimes in the diagonal conductivity $\sigma_{x x}$, stated above in (i)-(iv). It explains why the square-root exponent (corresponding to a $1 \mathrm{D}$ system) appears in the VRH conductivity $\sigma_{x x}$ of a 2D system. The tunneling-conductance oscillations in a two coupled Hall bars observed in [7] display a standard IQHE behavior which can not be understood as a tunneling between the two counter-propagating edge states. The puzzle resolves naturally in our model. A Hall line tunnel-junction couples Landau orbits across the junction which does not change the hopping mechanism of the model which remains basically the same as in the bulk of the sample. It is also worthy to note that the peak-split shape in Fig. 4, $a$ is typical for the IQHE conductors with the high mobility of electrons. The quantum term (2) in the $\sigma_{x x}$ plays an important role in our theory. This term is of the same origin as that in the conductivity across the layers in organic conductors $[6,17,18]$. The principal new point of the model is that Eqs. (1), (2) incorporate a localization into the description. The approach is open for a more specific models of localization, such, as models for the levitation of extended states [19]. This effect would be considered within the above model elsewhere.

The work was supported by INTAS, project INTAS-01-0791. The author is deeply gratitude to P. Fulde, J. Wosnitza, M. Kartsovnik, W. Biberacher, I.Ya. Polishchuk, I.V. Krive, A.M. Kosevich, A.A. Slutskin and participants of the theoretical seminar at FTINT in Kharkov for discussions and to S. Flach for the hospitality at MPIPKS in Dresden.

1. K. von Klitzing, G. Dorda, and M. Pepper, Phys. Rev. Lett. 45, 494 (1980).

2. R.B. Laughlin, Phys. Rev. B23, 5632 (1981); The Quantum Hall Effect, R.E. Prange and S.M. Girvin (eds.), Springer (1987).

3. B. Huckestein, Rev. Mod. Phys. 67, 357 (1995). 
4. F. Hohls, U. Zeitler, and R.J. Haug, Phys. Rev. Lett. 88, 036802 (2002).

5. D. Yashioka, The Quantum Hall Effect, Springer (2000).

6. V.M. Gvozdikov, Phys. Rev. B70, 085113 (2004).

7. I. Yang, W. Kang, K.W. Baldwin, L.N. Pfeiffer, and K.W. West, Phys. Rev. Lett. 92, 056802 (2004).

8. A.A. Abrikosov, Fundamentals of Theory of Metals, North-Holland, Amsterdam (1988).

9. A. Briggs, Y. Guldner, J.P. Vieren, M. Voos, J.P. Hirtz, and M. Razeghi, Phys. Rev. B27, 6549 (1983).

10. D.G. Polyakov and B.I. Shklovskii, Phys. Rev. B48, 11167 (1993); Phys. Rev. Lett. 70, 3796 (1993).

11. D. Shahar, D.C. Tsui, M. Shayegan, E. Shimshoni, and S.L. Sondhi, Phys. Rev. Lett. 79, 479 (1997).

12. N.F. Mott, J. Non-Cryst. Solids 1, 1 (1968); N.F. Mott and E.A. Davis, Electronic Processes in Non-Crystalline Materials, Clarendon, Oxford (1979).
13. M. Furlan, Phys. Rev. B57, 14818 (1998).

14. B.P. Dolan, Phys. Rev. B62, 10287 (2000).

15. J. Oswald, G. Span, and F. Kuchar, Phys. Rev. B58, 15401 (1998).

16. T. Maniv and I.D. Vagner, Phys, Rev. B38, 6301 (1988); P.D. Grigoriev, JETP 92, 1090 (2001); T. Champel, Phys. Rev. B64, 054407 (2001); V.M. Gvozdikov, A.G.M. Jansen, D.A. Pesin, I.D. Vagner, and P. Wyder, Phys. Rev. B68, 155107 (2003).

17. P.D. Grigoriev, Phys. Rev. B67, 144401 (2003).

18. T. Champel and V.P. Mineev, Phys. Rev. B66, 195111 (2002).

19. D.E. Khmelnitskii, Phys. Lett. A106, 182 (1984); R.B. Laughlin, Phys. Rev. Lett. 52, 2304 (1984), F.D.M. Haldane and K. Yang, Phys. Rev. Lett. 78, 298 (1997). 